\title{
Rotating string solutions: AdS/CFT duality in non-supersymmetric sectors
}

\author{
S. Frolov ${ }^{a, *}$ and A.A. Tseytlin ${ }^{a, b, * *}$ \\ ${ }^{a}$ Department of Physics, The Ohio State University, Columbus, OH 43210, USA \\ ${ }^{b}$ Blackett Laboratory, Imperial College, London, SW7 2BZ, U.K.
}

\begin{abstract}
We find the minimal energy solution describing a folded closed string located at the center of $A d S_{5}$ and rotating simultaneously in two planes in $S^{5}$ with two arbitrary $S O(6)$ angular momenta $J_{1}$ and $J_{2}$. In the case when $J_{1}=J_{2}=J^{\prime}$ we observe the precise agreement between the leading coefficient in the large $J^{\prime}$ expansion of the energy of this solution and the minimal eigen-value of the 1-loop anomalous dimension matrix of the corresponding $\left[J^{\prime}, 0, J^{\prime}\right]$ SYM operators obtained by Beisert, Minahan, Staudacher and Zarembo in hep-th/0306139. We find also perfect agreement between string and SYM results in cases of states with unequal spins dual to $\left[J_{2}, J_{1}-J_{2}, J_{2}\right]$ operators. This represents a remarkable quantitative test of the AdS/CFT duality in a non-supersymmetric (and non-near-BPS) sector.
\end{abstract}

$06 / 03$

* Also at Steklov Mathematical Institute, Moscow.

** Also at Lebedev Physics Institute, Moscow. 


\section{Introduction}

Precise quantitative checks of the AdS/CFT correspondence in non-trivial, i.e. nonsupersymmetric, cases were largely non-existent until very recently. A remarkable progress was initiated in [1], which suggested the possibility of direct comparison of energies of $A d S_{5} \times S^{5}$ string expanded near a particular BPS state (represented by a point-like string running along a geodesic in $S^{5}$ ) with perturbative dimensions of the corresponding SYM operators.

Since the comparison in the BMN [1] case is done for near-BPS states, one may still wonder if exact numerical tests of the AdS/CFT duality can be carried out also in a "far from BPS" sectors, i.e. when one expands near a non-supersymmetric string solution. Remarkably, the two recent developments on the SYM [2, 3, 4, 4, 5] and the string [6,7] sides have converged to provide such non-trivial tests.

The adequate interpretation of the proposal of [1] as a special case of a semiclassical expansion of $A d S_{5} \times S^{5}$ string theory selecting a particular sector of states with large charges was suggested in [8] and developed in [9,10]. Given a classical string solution carrying some global charges (e.g., $S O(6)$ angular momenta) 1 and parametrized by some constants $\mathrm{w}_{i}$, its classical $A d S_{5}$ energy and angular momenta $J_{s}$ can be written as $E=$ $\sqrt{\lambda} \mathcal{E}\left(\mathrm{w}_{i}\right), J_{s}=\sqrt{\lambda} \mathcal{J}_{s}\left(\mathrm{w}_{i}\right)$, where $\sqrt{\lambda}$ is the effective string tension (or 't Hooft coupling of the dual gauge theory). In the simplest and most interesting cases, some components of $J_{s}$ may be equal, so let us assume for simplicity that there is just one relevant parameter w and one value of $J$ and $J=\sqrt{\lambda}$ w. Then the energy can be written as $E=E(J, \sqrt{\lambda})$. The key assumptions that eventually allow one to compare to SYM theory are: (i) $\mathrm{w} \ll 1$, i.e. $g_{\text {eff }} \equiv \frac{1}{\mathrm{w}^{2}}=\frac{\lambda}{J^{2}} \ll 1$, and (ii) $J \gg 1$.

In the semiclassical expansion in string theory $\sqrt{\lambda} \gg 1$, while in perturbative SYM theory $\lambda \ll 1$, but $\frac{\lambda}{J^{2}} \ll 1$ should be satisfied on both sides of the duality to allow for the comparison. In addition, the value of $J$ should be numerically large in both theories. The assumption (i) allows one to write the classical energy as $E_{0}=k_{0} J\left(1+k_{1} \frac{\lambda}{J^{2}}+\ldots\right)$, where $k_{0}=1$ or 2 in the cases of interest [6] and dots stand for higher powers of $\frac{\lambda}{J^{2}}$. Other coefficients $\left(k_{1}, \ldots\right)$ may, in general, be functions of ratios of unequal angular momenta (cf. [7]). This looks like an expansion in positive integer powers of $\lambda$ and would suggest a possibility of comparison to perturbative anomalous dimensions of the corresponding

1 More generally, semiclassical considerations and matching onto SYM operators may apply to a broader sector of states with large oscillation numbers [11, 8, 12]. 
operators on the SYM side, if not for the fact that quantum string sigma model corrections should a priori give non-trivial contributions to $E$. It is here where the second assumption of $J \gg 1$ comes into play: it turns out that (due to underlying supersymmetry of the $A d S_{5} \times S^{5}$ superstring theory) quantum sigma model corrections to the above classical expression for the energy are suppressed by powers of $\frac{1}{J}$.

In more detail, on general grounds, the $\ell$-loop sigma model correction to the energy should have the form $E_{\ell}=\frac{1}{(\sqrt{\lambda})^{\ell-1}} \mathcal{E}_{\ell}(\mathrm{w})$. Assuming that $\mathcal{E}_{\ell}=\frac{c_{\ell}}{\mathrm{w}^{\lambda+1}}+\ldots$ for $\mathrm{w} \gg 1$ we would get $E_{\ell}=\frac{1}{J^{\ell-1}}\left(c_{\ell} \frac{\lambda}{J^{2}}+\ldots\right)$. In particular, $E_{1}=c_{1} \frac{\lambda}{J^{2}}+\ldots$. This is indeed what happens in the non-trivial three-spin example [6] as was shown explicitly at the 1-loop order in [7]. Then $E=E_{0}+E_{1}+\ldots=k_{0} J\left[1+k_{1} \frac{\lambda}{J^{2}}\left(1+\frac{c_{1}^{\prime}}{J}+\ldots\right)+\ldots\right], c_{1}^{\prime}=\frac{c_{1}}{k_{0} k_{1}}$. Assuming that string sigma model corrections to the classical energy are indeed suppressed in the large $J$ limit, one could then expect to match the terms in the expansion of the classical energy $E_{0}=k_{0}\left(J+k_{1} \frac{\lambda}{J}+k_{2} \frac{\lambda}{J^{3}}+\ldots\right)$ to the canonical dimension, one-loop anomalous dimension, two-loop anomalous dimension, etc., of the corresponding SYM operators with $J \gg 1$.

That sounds very much like the BMN story (cf. [9] and [13]), where similar discussion applied to excited states near a BPS state. Here instead we are considering the classical energy of a non-BPS "ground state" which is "far away" from BPS ground state of BMN. Matching to SYM anomalous dimensions would be already a non-trivial test of the AdS/CFT duality. One could then try to match also small excitations near a given non-BPS ground state.

Examples of simple rotating multiple-spin string solutions for which the above considerations should apply were recently constructed in [6,7]. These circular string solutions have two equal angular momenta $J_{1}=J_{2}=J^{\prime}$ in the two orthogonal planes, and the center of mass of the string is rotating in the third plane with angular momentum $J_{3}=J$. The solution with $J_{3}=0$ turns out to be unstable (for a similar reason why a closed string wound around large circle of a sphere is unstable). Adding extra angular momentum stabilizes the solution under small perturbations provided $J \geq \frac{2}{3} J^{\prime}$ [7]. 2

Similar semiclassical string states with $S O(6)$ spins $\left(J_{1}, J_{2}, J_{3}\right)$ should correspond to particular SYM scalar operators $\operatorname{tr}\left(X^{J_{1}} Y^{J_{2}} Z^{J_{3}}\right)+\ldots(X, Y, Z$ are three complex combinations of 6 real adjoint scalars) in irreducible $S U(4)$ representations with Dynkin labels $\left[J_{2}-J_{3}, J_{1}-J_{2}, J_{2}+J_{3}\right]$ (assuming for definiteness that $J_{1} \geq J_{2} \geq J_{3}$ ). To compare

2 A possible interpretation of why stabilization happens is that adding extra orbital motion provides a centrifugal force that compensates for the contraction caused by string tension. 
the string expressions in [7] with SYM theory one thus needs to know the eigen-values of perturbative anomalous dimension matrix of such operators for large values of $J_{i}$.

Progress in this direction was achieved very recently in [5] in the special case of $J_{3}=0$ : the eigen-values of the planar 1-loop anomalous dimension matrix for the scalar SYM operators $\operatorname{tr}\left(X^{J_{1}} Y^{J_{2}}\right)+\ldots$ were computed explicitly using integrable spin chain and dilatation operator technique, building on the previous work of [2, 4]. In the special case of $J_{1}=J_{2}=J^{\prime}$, i.e. $\left[J^{\prime}, 0, J^{\prime}\right]$ operators, it was found [5] that there exists a 1-loop anomalous dimension eigen-value that matches precisely onto the string theory prediction for the classical energy of a circular two-spin solution [6]. Moreover, the (real) energies of fluctuation modes near this solution [6] also have precise counterparts among gauge theory eigen-values [5].

A puzzling feature of this agreement, however, is that the two-spin circular string solution of [6] is unstable, i.e. there is a tachyonic fluctuation mode (not seen on gauge theory side [5]). One may wonder why the comparison should work at all for an unstable string solution. A possible explanation is that the matching actually works for a more general stable solution with $J_{3}>\frac{2}{3} J^{\prime}$, and for some reason the resulting expressions (for the energies of the ground state as well as fluctuation modes) on both sides of the duality admit analytic continuation to the region $J_{3}=0$. The analytic continuation should not work for the would-be tachyonic mode (whose energy and thus anomalous dimension would become imaginary) and that is why it was not seen in [5].

Another surprising result of [5] is the existence of $\left[J^{\prime}, 0, J^{\prime}\right]$ eigenvalue lower that the one corresponding to the circular solution of [6]. Believing in AdS/CFT, this suggests the existence of a different two-spin string solution in the sector of states with $J_{1}=J_{2}$ that has energy lower than the circular solution.

Our aim here is to show that such solution does exist and describes folded closed string rotating simultaneously in the two orthogonal planes with its center of mass positioned at a fixed point of $S^{5}$ (which is a generalisation of the single-spin solution in [8]). We find that the first subleading term in the energy of this solution $E=2 J^{\prime}\left(1+k_{1} \frac{\lambda}{J^{\prime 2}}+\ldots\right)$ has indeed exactly the same coefficient as the corresponding minimal 1-loop anomalous dimension on the SYM side! This provides a remarkable test of the above AdS/CFT duality considerations.

The general folded string solution we shall consider will have two arbitrary angular momenta $J_{1}, J_{2}$. We shall find its energy by expanding in $\frac{\lambda}{\left(J_{1}+J_{2}\right)^{2}} \ll 1$ and $\frac{J_{2}}{J_{1}+J_{2}} \ll 1$, and 
observe again that the first subleading term in $E$ matches precisely with the corresponding 1-loop anomalous dimension eigenvalue for the operator $\left[J_{2}, J_{1}-J_{2}, J_{2}\right]$ obtained in [5].

It should be noted that, as in many similar duality contexts, easy-to-find classical string energy expressions provide predictions for all-loop anomalous dimension expressions on the SYM side (which are rather complicated to find already at the 1-loop order). Such precise matching between the string and SYM results as found in [5] and here which works for different string states suggests a more direct equivalence between an effective anomalous dimension Hamiltonian (or a kind of dilatation operator) on the SYM side and the classical string action (both expanded in $\frac{\lambda}{J^{2}} \ll 1$ and for $J \ll 1$ ).

While the two-spin folded string solution we present below is a direct generalization of the single-spin solution in [8, 14], it should be noted that the two-spin case turns out to be better suited for comparison with the SYM theory than the single-spin case - in the latter the classical energy has the following expansion $[8]: E=J+\frac{2 \sqrt{\lambda}}{\pi}+O\left(\frac{1}{J}\right)$. The leading correction here is much larger than its counterpart $\frac{\lambda}{J}$ in the two-spin case (assuming $\frac{\lambda}{J^{2}} \ll 1$ ), and thus its direct comparison to SYM theory is problematic (it should also receive corrections in string sigma model loop expansion). The point is that in the single-spin sector the minimal-energy state is represented by the BMN point-like string dual to the BPS gauge-theory operator $\operatorname{tr} Z^{J}$, while other states having higher energy should be represented by operators with extra zero R-charge insertions like $\operatorname{tr}\left(Z^{J} X \bar{X} \ldots\right)$ or $\operatorname{tr}\left[Z^{J}\left(F_{m n}\right)^{2} \ldots\right]$. At the same time, the folded string solution appears to represent the minimal energy configuration in the two-spin sector (where there is apparently no BPS state) and that is why it can be matched onto the minimal dimension operators like $\operatorname{tr}\left(X^{J_{1}} Y^{J_{2}}\right)+\ldots$

\section{Two-spin string solution in $A d S_{5} \times S^{5}$}

Let us start with specifying our notation. The bosonic part of the $A d S_{5} \times S^{5}$ string action is

$$
I=-\frac{\sqrt{\lambda}}{4 \pi} \int d^{2} \xi\left[G_{m n}^{\left(A d S_{5}\right)}(x) \partial_{a} x^{m} \partial^{a} x^{n}+G_{p q}^{\left(S^{5}\right)}(y) \partial_{a} y^{p} \partial^{a} y^{q}\right], \quad \sqrt{\lambda} \equiv \frac{R^{2}}{\alpha^{\prime}}
$$

We shall use the following explicit parametrization of the unit-radius metric on $S^{5}$ :

$$
\left(d s^{2}\right)_{S^{5}}=d \gamma^{2}+\cos ^{2} \gamma d \varphi_{3}^{2}+\sin ^{2} \gamma\left(d \psi^{2}+\cos ^{2} \psi d \varphi_{1}^{2}+\sin ^{2} \psi d \varphi_{2}^{2}\right)
$$


It will be convenient to choose the range of $\psi$ to be the interval $[-\pi, \pi]$. This metric has three translational isometries in $\varphi_{i}$, so that in addition to the three $A d S_{5}$ integrals of motion, a general solution should also have the following three integrals of motion depending on the $S^{5}$ part of the action:

$$
\begin{gathered}
J_{3}=\sqrt{\lambda} \int_{0}^{2 \pi} \frac{d \sigma}{2 \pi} \cos ^{2} \gamma \partial_{0} \varphi_{3} \equiv \sqrt{\lambda} \mathcal{J}_{3}, \\
J_{1}=\sqrt{\lambda} \int_{0}^{2 \pi} \frac{d \sigma}{2 \pi} \sin ^{2} \gamma \cos ^{2} \psi \partial_{0} \varphi_{1} \equiv \sqrt{\lambda} \mathcal{J}_{1}, \\
J_{2}=\sqrt{\lambda} \int_{0}^{2 \pi} \frac{d \sigma}{2 \pi} \sin ^{2} \gamma \sin ^{2} \psi \partial_{0} \varphi_{2} \equiv \sqrt{\lambda} \mathcal{J}_{2} .
\end{gathered}
$$

Let us look for a classical solution describing a closed folded string located at the center $\rho=0$ of $A d S_{5}$ and at fixed value of the $S^{5}$ angle $\gamma=\frac{\pi}{2}$, rotating within $S^{3}$ part of $S^{5}$ with arbitrary frequencies $\mathrm{w}_{1}$ and $\mathrm{w}_{2}$. A natural ansatz for such a solution is

$$
t=\kappa \tau, \quad \rho=0, \quad \gamma=\frac{\pi}{2}, \quad \varphi_{3}=0, \quad \varphi_{1}=\mathrm{w}_{1} \tau, \quad \varphi_{2}=\mathrm{w}_{2} \tau, \quad \psi=\psi(\sigma)
$$

where $\kappa, \mathrm{w}_{1}, \mathrm{w}_{2}=$ const. Such solution will have $J_{3}=0, J_{1} \neq 0, J_{2} \neq 0$. The energy is

$$
E=\sqrt{\lambda} \kappa=E\left(J_{1}, J_{2}, \lambda\right)
$$

The special case of $\mathrm{w}_{1}=0\left(J_{1}=0\right)$ will give the single-spin folded string solution considered in [8]. One of our aims will be to find a minimal energy solution in the sector of semiclassical string configurations with $J_{1}=J_{2}$.

We will assume without loss of generality that $\mathrm{w}_{2} \geq \mathrm{w}_{1}$ and introduce

$$
\mathrm{w}_{21}^{2} \equiv \mathrm{w}_{2}^{2}-\mathrm{w}_{1}^{2} \geq 0
$$

The string equations of motion for the angles in the conformal gauge then lead to the following equation for $\psi$

$$
\psi^{\prime \prime}+\frac{1}{2} \mathrm{w}_{21}^{2} \sin 2 \psi=0
$$

Integrating this once, we get

$$
\psi^{\prime 2}=\mathrm{w}_{21}^{2}\left(\sin ^{2} \psi_{0}-\sin ^{2} \psi\right)+\mathrm{k}^{2}
$$


where the integration constant $\psi_{0}$ will be determining the length of a folded string as in 8]. We have introduced an (integer) parameter $\mathrm{k}$ to include also the case when $\mathrm{w}_{1}=\mathrm{w}_{2}$. The conformal gauge constraints lead to the following relation between $\kappa, x_{0}$ and $\mathrm{w}_{i}$

$$
\kappa^{2}=\psi^{\prime 2}+\mathrm{w}_{1}^{2} \cos ^{2} \psi+\mathrm{w}_{2}^{2} \sin ^{2} \psi=\mathrm{w}_{2}^{2} \sin ^{2} \psi_{0}+\mathrm{w}_{1}^{2} \cos ^{2} \psi_{0}+\mathrm{k}^{2} .
$$

There are two distinct solutions, the circular (C) one [6] and the new folded (F) one (where $\mathrm{k}=0)$ : 3

$$
\begin{gathered}
C: \quad \mathrm{w}_{1}=\mathrm{w}_{2}=\mathrm{w}, \quad \psi=\mathrm{k} \sigma, \quad \kappa^{2}=\mathrm{w}^{2}+\mathrm{k}^{2}, \quad \mathrm{k}=1,2, \ldots, \quad \\
F: \quad \mathrm{w}_{1} \neq \mathrm{w}_{2}, \quad \psi=\psi(\sigma), \quad-\psi_{0} \leq \psi(\sigma) \leq \psi_{0}, \quad \kappa^{2}=\mathrm{w}_{2}^{2} \sin ^{2} \psi_{0}+\mathrm{w}_{1}^{2} \cos ^{2} \psi_{0} .
\end{gathered}
$$

For the single-fold solution the periodicity in $\sigma$ implies the following condition on the parameters:

$$
2 \pi=\int_{0}^{2 \pi} d \sigma=4 \int_{0}^{\psi_{0}} \frac{d \psi}{\mathrm{w}_{21} \sqrt{\sin ^{2} \psi_{0}-\sin ^{2} \psi}} .
$$

The angular momenta are

$$
\begin{aligned}
& \mathcal{J}_{1}=\mathrm{w}_{1} \int_{0}^{2 \pi} \frac{d \sigma}{2 \pi} \cos ^{2} \psi=\frac{2 \mathrm{w}_{1}}{\pi \mathrm{w}_{21}} \int_{0}^{\psi_{0}} \frac{\cos ^{2} \psi d \psi}{\sqrt{\sin ^{2} \psi_{0}-\sin ^{2} \psi}}, \\
& \mathcal{J}_{2}=\mathrm{w}_{2} \int_{0}^{2 \pi} \frac{d \sigma}{2 \pi} \sin ^{2} \psi=\frac{2 \mathrm{w}_{2}}{\pi \mathrm{w}_{21}} \int_{0}^{\psi_{0}} \frac{\sin ^{2} \psi d \psi}{\sqrt{\sin ^{2} \psi_{0}-\sin ^{2} \psi}} .
\end{aligned}
$$

Note the following relation

$$
\frac{\mathcal{J}_{1}}{\mathrm{w}_{1}}+\frac{\mathcal{J}_{2}}{\mathrm{w}_{2}}=1
$$

Computing the integrals, we get the following expressions in terms of the hypergeometric functions

$$
\begin{gathered}
\mathrm{w}_{21}={ }_{2} F_{1}\left(\frac{1}{2}, \frac{1}{2} ; 1 ; x_{0}\right), \quad x_{0} \equiv \sin ^{2} \psi_{0} \\
\mathcal{J}_{1}=\frac{\mathrm{w}_{1}}{\mathrm{w}_{21}}{ }_{2} F_{1}\left(-\frac{1}{2}, \frac{1}{2} ; 1 ; x_{0}\right), \quad \mathcal{J}_{2}=\frac{\mathrm{w}_{2} x_{0}}{2 \mathrm{w}_{21}}{ }_{2} F_{1}\left(\frac{1}{2}, \frac{3}{2} ; 2 ; x_{0}\right) .
\end{gathered}
$$

These relations can be used to find the dependence of $\mathrm{w}_{i}$ and $\psi_{0}$ on the angular momenta $J_{1}, J_{2}$, and then, using (2.7), 2.10) one can find the dependence of the energy on the angular momenta.

3 As in the single-spin case 8 , the solution with both $\mathrm{w}_{21}$ and $\mathrm{k}$ being non-zero (i.e. a generalization of the circular string solution) is expected to be unstable. 


\section{Sector of equal spins}

Let us now consider the case

$$
J_{1}=J_{2}=J^{\prime} .
$$

In the circular solution case we get $($ here $\mathrm{k}=1)$ [6]

$$
E=2 J^{\prime} \sqrt{1+\frac{\lambda}{\left(2 J^{\prime}\right)^{2}}} .
$$

In the case of the folded string solution, which happens to have lower (minimal) energy in this sector, the expression for the energy $E=E\left(J^{\prime}, \lambda\right)$ can be found in expansion in powers of

$$
\frac{1}{\mathcal{J}^{2}}=\frac{\lambda}{J^{\prime 2}} \ll 1 \text {. }
$$

Solving (2.16), (2.17) and (2.18), we find

$$
\begin{gathered}
x_{0}=0.826115-\frac{0.0784101}{\mathcal{J}^{2}}+\frac{0.00697882}{\mathcal{J}^{4}}+\ldots \\
\mathrm{w}_{1}=2 \mathcal{J}-\frac{0.272922}{\mathcal{J}}+\frac{0.0858257}{\mathcal{J}^{3}}+\ldots, \quad \mathrm{w}_{2}=2 \mathcal{J}+\frac{0.272922}{\mathcal{J}}-\frac{0.0113391}{\mathcal{J}^{3}}+\ldots, \\
E=2 J^{\prime}+k_{1} \frac{\lambda}{4 J^{\prime}}-k_{2} \frac{\lambda^{2}}{64 J^{\prime 3}}+\ldots \\
k_{1}=0.712032, \quad k_{2}=1.69878 .
\end{gathered}
$$

For comparison, for the circular string solution (3.1) we find

$$
E=2 J^{\prime}+\frac{\lambda}{4 J^{\prime}}-\frac{\lambda^{2}}{64 J^{\prime 3}}+\ldots .
$$

While the numerical coefficients in (3.4) are of order 1 as in (3.6), the energy of the folded string solution (3.4) is clearly lower than the energy of the circular solution for a given value of the angular momentum $J^{\prime} \gg \sqrt{\lambda}$. The expression (3.4) applies to the single-fold solution; the result for the $\mathrm{n}$-fold solution turns out to be the same but with $\lambda$ replaced by $\lambda n^{2}$, i.e.

$$
E=2 J^{\prime}+k_{1} \frac{\lambda \mathrm{n}^{2}}{4 J^{\prime}}-k_{2} \frac{\lambda^{2} \mathrm{n}^{4}}{64 J^{\prime 3}}+\ldots, \quad \mathrm{n}=1,2, \ldots .
$$

Similarly, one can generalize (3.6) by introducing the "winding number" $\mathrm{k}$ in (2.11) by $\lambda \rightarrow \lambda \mathrm{k}^{2}$ (see [7]). Thus the double-fold solution has higher energy than the singly-wound circular string, but still lower energy than the doubly-wound string, etc. 
As was found in [5], the subleading term in (3.6) can be matched precisely onto an eigen-state of 1-loop anomalous dimension operator for the scalar $\left[J^{\prime}, 0, J^{\prime}\right]$ operators on the SYM side. Ref. [5] discovered also a lower anomalous dimension eigen-value for which there was no apparent string theory counterpart. It was suggested in [5] that there should be a dual semiclassical solution which should then involve elliptic integrals in order to match the Bethe ansatz prediction for the anomalous dimension.

This is indeed what we find here: the minimal-eigenvalue gauge-theory operator should be dual to the minimal-energy two-spin folded string solution. Comparing the second term in $E$ in (3.4) with the result for the minimal value of the 1-loop anomalous dimension of the $\left[J^{\prime}, 0, J^{\prime}\right]$ scalar operator found in [5] we observe the exact agreement of the numerical coefficient $k_{1}$ with the coefficient in eq. (1.7) in [5]! !

The third term in (3.4) represents a prediction for the two-loop perturbative correction to the dimension of the SYM $\left[J^{\prime}, 0, J^{\prime}\right]$ scalar operator. It is plausible that this prediction can be verified by using the results of [15].

\section{Case of unequal spins}

If the spins $J_{1}, J_{2}$ are arbitrary, there are two interesting cases to consider. The first case corresponds to

$$
J_{2} \ll L, \quad L \equiv J_{1}+J_{2}, \quad \text { i.e. } \quad \alpha \equiv \frac{J_{2}}{L} \ll 1 .
$$

Such string states should be dual to SYM operators close to the BPS operator in the $\left[0, J_{1}, 0\right]$ representation $\left(J_{1} \gg J_{2}\right)$.

The second case is the one of almost equal spins, i.e.

$$
S \ll L, \quad S \equiv J_{1}-J_{2} .
$$

Such string states should be dual to SYM operators close to the operator in the $\left[J^{\prime}, 0, J^{\prime}\right]$ irrep.

4 Both numerical coefficients come from values of certain hypergeometric functions that can probably be matched directly. 


\section{1. $\alpha \ll 1$}

Solving (2.16), 2.17) and 2.18), and introducing $\mathcal{L}=\frac{L}{\sqrt{\lambda}}$, we find the following expansions up to the order $\alpha^{8}$

$$
\begin{gathered}
x_{0}=2 \alpha\left(1-\frac{\alpha}{4}-\frac{\alpha^{2}}{8}-\frac{11 \alpha^{3}}{128}-\frac{17 \alpha^{4}}{256}-\frac{55 \alpha^{5}}{1024}-\frac{179 \alpha^{6}}{4096}-\frac{9061 \alpha^{7}}{262144}\right) \\
-\frac{\alpha}{\mathcal{L}^{2}}\left(1-\frac{\alpha}{2}-\frac{\alpha^{2}}{4}-\frac{7 \alpha^{3}}{32}-\frac{119 \alpha^{4}}{512}-\frac{269 \alpha^{5}}{1024}-\frac{307 \alpha^{6}}{1024}-\frac{43165 \alpha^{7}}{32768}\right) \\
+\frac{3 \alpha}{4 \mathcal{L}^{4}}\left(1-\alpha-\frac{3 \alpha^{2}}{8}-\frac{5 \alpha^{3}}{16}-\frac{47 \alpha^{4}}{128}-\frac{63 \alpha^{5}}{128}-\frac{2799 \alpha^{6}}{4096}+\frac{39151 \alpha^{7}}{32768}\right)+\ldots \\
\mathrm{w}_{1}=\mathcal{L}-\frac{\alpha}{2 \mathcal{L}}\left(1+\alpha+\frac{9 \alpha^{2}}{8}+\frac{21 \alpha^{3}}{16}+\frac{795 \alpha^{4}}{512}+\frac{945 \alpha^{5}}{512}+\frac{2247 \alpha^{6}}{1024}+\frac{26589 \alpha^{7}}{16384}\right) \\
\frac{3 \alpha}{8 \mathcal{L}^{3}}\left(1+\frac{4 \alpha}{3}+\frac{25 \alpha^{2}}{12}+\frac{13 \alpha^{3}}{4}+\frac{1267 \alpha^{4}}{256}+\frac{235 \alpha^{5}}{32}+\frac{21789 \alpha^{6}}{2048}+\frac{564453 \alpha^{7}}{32768}\right)+\ldots \\
\mathrm{w}_{2}=\mathcal{L}+\frac{1}{2 \mathcal{L}}\left(1+\frac{\alpha^{2}}{8}+\frac{3 \alpha^{3}}{16}+\frac{123 \alpha^{4}}{512}+\frac{75 \alpha^{5}}{256}+\frac{357 \alpha^{6}}{1024}-\frac{9363 \alpha^{7}}{16384}-\frac{26589 \alpha^{8}}{16384}\right) \\
\frac{1}{8 \mathcal{L}^{3}}\left(1-\alpha-\frac{\alpha^{2}}{4}+\frac{\alpha^{3}}{4}+\frac{219 \alpha^{4}}{256}+\frac{429 \alpha^{5}}{256}+\frac{5721 \alpha^{6}}{2048}+\frac{479271 \alpha^{7}}{32768}-\frac{6662229 \alpha^{8}}{131072}\right)+\ldots
\end{gathered}
$$

The resulting expression for the energy is

$$
\begin{gathered}
E=L+m_{1} \frac{\alpha \lambda}{2 L}-m_{2} \frac{\alpha \lambda^{2}}{8 L^{3}}+\ldots \\
m_{1}=1+\frac{\alpha}{2}+\frac{3 \alpha^{2}}{8}+\frac{21 \alpha^{3}}{64}+\frac{159 \alpha^{4}}{512}+\frac{315 \alpha^{5}}{1024}+\frac{321 \alpha^{6}}{1024}+\ldots \\
m_{2}=1+2 \alpha+\frac{9 \alpha^{2}}{4}+\frac{21 \alpha^{3}}{8}+\frac{807 \alpha^{4}}{256}+\frac{123 \alpha^{5}}{32}+\frac{9663 \alpha^{6}}{2048}+\ldots
\end{gathered}
$$

Remarkably, the expression for the coefficient $m_{1}$ (4.5) in the energy is exactly the same as the corresponding 1-loop anomalous dimension eigenvalue in eq. (2.21) in [5]! The $m_{2}$-term in (4.4) is a prediction for the two-loop anomalous dimension. The first terms (one) in $m_{1}$ and $m_{2}$ are consistent with the expansion of the pp-wave [16] spectrum [17] at small $\alpha$. 


\section{2. $S \ll L$}

Solving (2.16), (2.17) and (2.18), and introducing $\beta=\frac{S}{L}$, we find up to the order $\beta^{3}$

$$
\begin{gathered}
x_{0}=0.83-0.57 \beta-0.37 \beta^{2}+0.19 \beta^{3} \\
-\frac{0.31}{\mathcal{L}^{2}}\left(1-1.85 \beta^{2}+1.74 \beta^{3}\right)+\frac{0.11}{\mathcal{L}^{4}}\left(1+3.07 \beta-8.55 \beta^{2}+11.46 \beta^{3}\right)+\ldots \\
\mathrm{w}_{1}=\mathcal{L}-\frac{0.55}{\mathcal{L}}\left(1-2.30 \beta+3.16 \beta^{2}-4.46 \beta^{3}\right)+\frac{0.69}{\mathcal{L}^{3}}\left(1-3.61 \beta+8.67 \beta^{2}-18.46 \beta^{3}\right)+\ldots, \\
\mathrm{w}_{2}=\mathcal{L}+\frac{0.56}{\mathcal{L}}\left(1-0.30 \beta+0.56 \beta^{2}-0.76 \beta^{3}\right)-\frac{0.09}{\mathcal{L}^{3}}\left(1-1.61 \beta+7.72 \beta^{2}-17.60 \beta^{3}\right)+\ldots,
\end{gathered}
$$

and the energy is

$$
\begin{gathered}
E=L+d_{1} \frac{\lambda}{L}+d_{2} \frac{\lambda^{2}}{L^{3}}+\ldots, \\
d_{1}=0.356016-0.545845 \beta+0.356016 \beta^{2}-0.336751 \beta^{3}+\ldots, \\
d_{2}=-0.212347+0.582988 \beta-0.931684 \beta^{2}+1.42221 \beta^{3}+\ldots .
\end{gathered}
$$

The first two terms in the expression for $d_{1}$ coincide with the corresponding 1-loop anomalous dimension obtained in Section 2 of [5], i.e. we find again a perfect agreement. The expression for $d_{2}$ represents the string theory prediction for the 2-loop perturbative anomalous dimension.

\section{Concluding remarks}

There are several possible generalizations. We can try to generalize the above 2-spin folded string solution by introducing one extra angular momentum component $J_{3}$ adding the rotation in $\varphi_{3}$ angle. In contrast to the circular solution case in [9,7] where we could keep the angle $\gamma$ constant, here it should be made $\sigma$-dependent:

$$
t=\kappa \tau, \quad \varphi_{1}=\mathrm{w}_{1} \tau, \quad \varphi_{2}=\mathrm{w}_{2} \tau, \quad \varphi_{3}=\mathrm{w}_{3} \tau, \quad \gamma=\gamma(\sigma), \quad \psi=\psi(\sigma)
$$

The resulting equation for $\psi$ becomes (cf. (2.8))

$$
\left(\sin ^{2} \gamma \psi^{\prime}\right)^{\prime}+\frac{1}{2}\left(\mathrm{w}_{2}^{2}-\mathrm{w}_{1}^{2}\right) \sin ^{2} \gamma \sin 2 \psi=0
$$

while the equation for $\gamma$ follows from the conformal gauge constraint (cf. 2.10)

$$
\gamma^{\prime 2}+\mathrm{w}_{3}^{2} \cos ^{2} \gamma+\sin ^{2} \gamma\left(\psi^{\prime 2}+\mathrm{w}_{1}^{2} \cos ^{2} \psi+\mathrm{w}_{2}^{2} \sin ^{2} \psi\right)=\kappa^{2}
$$


We leave the analysis of this case (which is related to an integrable model) for the future work [18]. One can also consider similar minimal-energy two-spin solutions in $A d S_{5}$, generalizing single-spin solution considered in [8]. The challenge then will be to find the SYM predictions for the corresponding anomalous dimensions to allow for a comparison.

\section{Acknowledgements}

We are grateful to J. Russo and K. Zarembo for useful discussions. We also thank N. Beisert, J. Minahan, M. Staudacher and K. Zarembo for informing us about ref. [5] prior to publication. This work was supported by the DOE grant DE-FG02-91ER40690. The work of A.T. was also supported in part by the PPARC SPG 00613 and INTAS 99-1590 grants and the Royal Society Wolfson award. 


\section{References}

[1] D. Berenstein, J. Maldacena and H. Nastase, "Strings in flat space and pp waves from N = 4 super Yang Mills," JHEP 0204, 013 (2002) hep-th/0202021.

[2] N. Beisert, C. Kristjansen, J. Plefka, G. W. Semenoff and M. Staudacher, "BMN correlators and operator mixing in $\mathrm{N}=4$ super Yang-Mills theory," Nucl. Phys. B 650, 125 (2003) hep-th/0208178].

[3] N. Beisert, C. Kristjansen, J. Plefka and M. Staudacher, "BMN gauge theory as a quantum mechanical system," Phys. Lett. B 558, 229 (2003) [hep-th/0212269.

[4] J. A. Minahan and K. Zarembo, "The Bethe-ansatz for $\mathrm{N}=4$ super Yang-Mills," JHEP 0303, 013 (2003) hep-th/0212208.

[5] N. Beisert, J. Minahan, M. Staudacher and K. Zarembo, "Stringing Spins and Spinning Strings," hep-th/0306139.

[6] S. Frolov and A. A. Tseytlin, "Multi-spin string solutions in $A d S_{5} \times S^{5}$," hepth/0304255.

[7] S. Frolov and A. A. Tseytlin, "Quantizing three-spin string solution in $A d S_{5} \times S^{5}$, hep-th/0306130.

[8] S. S. Gubser, I. R. Klebanov and A. M. Polyakov, "A semi-classical limit of the gauge/string correspondence," Nucl. Phys. B 636, 99 (2002) [hep-th/0204051].

[9] S. Frolov and A. A. Tseytlin, "Semiclassical quantization of rotating superstring in $A d S_{5} \times S^{5}, "$ JHEP 0206, 007 (2002) [hep-th/0204226].

[10] A. A. Tseytlin, "Semiclassical quantization of superstrings: $A d S_{5} \times S^{5}$ and beyond," Int. J. Mod. Phys. A 18, 981 (2003) hep-th/0209116. "On semiclassical approximation and spinning string vertex operators in $A d S_{5} \times S^{5}$," hep-th/0304139.

[11] A. M. Polyakov, "Gauge fields and space-time," Int. J. Mod. Phys. A 17S1, 119 (2002) hep-th/0110196.

[12] J. A. Minahan, "Circular semiclassical string solutions on $A d S_{5} \times S^{5}$," Nucl. Phys. B 648, 203 (2003) hep-th/0209047.

[13] C. Kristjansen, J. Plefka, G. W. Semenoff and M. Staudacher, "A new double-scaling limit of $\mathrm{N}=4$ super Yang-Mills theory and PP-wave strings," Nucl. Phys. B 643, 3 (2002) hep-th/0205033]. D. J. Gross, A. Mikhailov and R. Roiban, "Operators with large R charge in N = 4 Yang-Mills theory," Annals Phys. 301, 31 (2002) hepth/0205066]. N. R. Constable, D. Z. Freedman, M. Headrick, S. Minwalla, L. Motl, A. Postnikov and W. Skiba, "PP-wave string interactions from perturbative YangMills theory," JHEP 0207, 017 (2002) hep-th/0205089.

[14] J. G. Russo, "Anomalous dimensions in gauge theories from rotating strings in $A d S_{5} \times$ $S^{5}, "$ JHEP 0206, 038 (2002) hep-th/0205244.

[15] N. Beisert, C. Kristjansen and M. Staudacher, "The dilatation operator of $\mathrm{N}=4$ super Yang-Mills theory," hep-th/0303060. 
[16] M. Blau, J. Figueroa-O'Farrill, C. Hull and G. Papadopoulos, "A new maximally supersymmetric background of IIB superstring theory," JHEP 0201, 047 (2002) hepth/0110242.

[17] R. R. Metsaev, "Type IIB Green-Schwarz superstring in plane wave Ramond-Ramond background," Nucl. Phys. B 625, 70 (2002) hep-th/0112044. R. R. Metsaev and A. A. Tseytlin, "Exactly solvable model of superstring in plane wave Ramond-Ramond background," Phys. Rev. D 65, 126004 (2002) [hep-th/0202109].

[18] G. Arutyunov, S. Frolov, J.G. Russo and A. A. Tseytlin, work in progress. 